\title{
Localization for random block operators
}

\author{
Martin Gebert and Peter Müller
}

\begin{abstract}
We continue the investigations of Kirsch, Metzger and the second-named author [J. Stat. Phys. 143, 1035-1054 (2011)] on spectral properties of a certain type of random block operators. In particular, we establish an alternative version of a Wegner estimate and an improved result on Lifschitz tails at the internal band edges. Using these ingredients and the bootstrap multi-scale analysis, we also prove dynamical localization in a neighbourhood of the internal band edges.
\end{abstract}

\section{The model and its basic properties}

Random block operators arise in several different fields of Theoretical Physics. In this paper we are concerned with those that are relevant to mesoscopic disordered systems such as dirty superconductors. In this context, block operators are used to describe quasi-particle excitations within the self-consistent Bogoliubov-de Gennes equations. It turns out that such block operators fall in 10 different symmetry classes [AZ97]. As in the previous paper [KMM11, we will consider one particular symmetry class, class $C 1$, and refer to KMM11. for further discussions and motivations.

Given some Hilbert space $\mathcal{H}$, we write $\mathcal{L}(\mathcal{H})$ for the Banach space of all bounded linear operators from $\mathcal{H}$ into itself. In this paper we are concerned with the Hilbert space $\mathcal{H}^{2}:=\ell^{2}\left(\mathbb{Z}^{d}\right) \oplus \ell^{2}\left(\mathbb{Z}^{d}\right)$, the direct sum of two Hilbert spaces of complex-valued, square-summable sequences indexed by the $d$ dimensional integers $\mathbb{Z}^{d}$. We also fix a probability space $(\Omega, \mathcal{F}, \mathbb{P})$ with corresponding expectation denoted by $\mathbb{E}$.

Definition 1.1. In this paper a random block operator is an operator-valued random variable

$$
\begin{aligned}
\Omega \longrightarrow \mathcal{L}\left(\mathcal{H}^{2}\right) \\
\mathbb{H}: \\
\omega \longmapsto \mathbb{H}^{\omega}:=\left(\begin{array}{cc}
H^{\omega} & B^{\omega} \\
B^{\omega} & -H^{\omega}
\end{array}\right)
\end{aligned}
$$

with the following three properties:

Version of 9 August 2012. 
(i) For $\mathbb{P}$-a.e. $\omega \in \Omega$ the operator $H^{\omega}:=H_{0}+V^{\omega} \in \mathcal{L}\left(l^{2}\left(\mathbb{Z}^{d}\right)\right)$ is the discrete random Schrödinger operator of the Anderson model. More precisely, $H_{0}$ stands for the negative discrete Laplacian on $\mathbb{Z}^{d}$, which is defined by

$$
\left(H_{0} \psi\right)(n):=-\sum_{m \in \mathbb{Z}^{d}:|m-n|=1}[\psi(m)-\psi(n)]
$$

for every $\psi \in \ell^{2}\left(\mathbb{Z}^{d}\right)$ and every $n \in \mathbb{Z}^{d}$. We always stick to the 1 -norm $|n|:=\sum_{j=1}^{d}\left|n_{j}\right|$ of $n=\left(n_{1}, \ldots, n_{d}\right) \in \mathbb{Z}^{d}$.

The random potential is induced by a given family $\left(\omega \mapsto V_{n}^{\omega}\right)_{n \in \mathbb{Z}^{d}}$ of i.i.d. real-valued random variables on $\Omega$ with single-site measure $\mu_{V}$ of compact support in $\mathbb{R}$. Thus, the multiplication operator given by

$$
\left(V^{\omega} \psi\right)(n):=V_{n}^{\omega} \psi(n)
$$

for every $\psi \in \ell^{2}\left(\mathbb{Z}^{d}\right)$ and every $n \in \mathbb{Z}^{d}$ is well-defined and bounded for $\mathbb{P}$-a.e. $\omega \in \Omega$. Also, $H^{\omega}$ is self-adjoint and bounded for $\mathbb{P}$-a.e. $\omega \in \Omega$.

(ii) For $\mathbb{P}$-a.e. $\omega \in \Omega$ the operator $B^{\omega} \in \mathcal{L}\left(\ell^{2}\left(\mathbb{Z}^{d}\right)\right)$ is the multiplication operator induced by the family $\left(\omega \mapsto B_{n}^{\omega}\right)_{n \in \mathbb{Z}^{d}}$ of i.i.d. real-valued random variables on $\Omega$ with single-site measure $\mu_{B}$ of compact support in $\mathbb{R}$.

(iii) The family of random variables $\left(V_{n}\right)_{n \in \mathbb{Z}^{d}}$ is independent of the family $\left(B_{n}\right)_{n \in \mathbb{Z}^{d}}$.

Remarks 1.2. (i) Conditions (i) and (ii) in Definition 1.1 imply that the random block operator $\mathbb{H}$ is $\mathbb{P}$-a.s. self-adjoint and bounded.

(ii) Block operators of the form (1.1) have a spectrum that is symmetric around 0, i.e. $E \in \mathbb{R}$ belongs to the spectrum $\sigma\left(\mathbb{H}^{\omega}\right)$, if and only if this is also true for $-E$ [KMM11, Lemma 2.3].

(iii) The random block operator $H$ is ergodic with respect to $\mathbb{Z}^{d}$ translations, see KMM11 for more details. Therefore, standard results imply the existence of a non-random closed set $\Sigma$ such that $\sigma(\mathbb{H})=\Sigma$ holds $\mathbb{P}$-a.s. K89, K08, CL90, PF92. This non-randomness also extends to the components in the Lebesgue decomposition of the spectrum.

In order to count eigenvalues we introduce a restriction of random block operators to bounded regions of space $\mathbb{Z}^{d}$. Given $L>0$ we write $\Lambda_{L}:=$ ] $-L / 2, L / 2\left[{ }^{d} \cap \mathbb{Z}^{d}\right.$ for the discrete cube of "length $L$ " about the origin and $\Lambda_{L}(n):=n+\Lambda_{L}$ for its shifted copy with centre $n \in \mathbb{Z}^{d}$.

Definition 1.3. Given a cube $\Lambda_{L} \subset \mathbb{Z}^{d}$, we define the finite-volume Hilbert space $\mathcal{H}_{L}^{2}:=\ell^{2}\left(\Lambda_{L}\right) \oplus \ell^{2}\left(\Lambda_{L}\right)$ and the finite-volume random block operator

$$
\begin{aligned}
& \Omega \longrightarrow \mathcal{L}\left(\mathcal{H}_{L}^{2}\right) \\
\mathbb{H}_{\Lambda_{L}} \equiv \mathbb{H}_{L}: & \omega \longmapsto \mathbb{H}_{L}^{\omega}:=\left(\begin{array}{cc}
H_{L}^{\omega} & B^{\omega} \\
B^{\omega} & -H_{L}^{\omega}
\end{array}\right),
\end{aligned}
$$

where $H_{L}:=H_{0, L}+V$ and $H_{0, L}$ is the discrete Laplacian on $\Lambda_{L}$ with simple boundary conditions. Its matrix entries are given by $H_{0, L}(n, m):=$ $\left\langle\delta_{n}, H_{0} \delta_{m}\right\rangle$ for $n, m \in \Lambda_{L}$, with $\left(\delta_{n}\right)_{n \in \mathbb{Z}^{d}}$ denoting the canonical basis and 
$\langle\cdot, \cdot\rangle$ the canonical scalar product of $\ell^{2}\left(\mathbb{Z}^{d}\right)$. The random multiplication operators $V$ and $B$ are restricted to $\ell^{2}\left(\Lambda_{L}\right)$ in the canonical way.

Remarks 1.4. (i) The operator $\mathbb{H}_{L}^{\omega}$ is well-defined, bounded and selfadjoint for $\mathbb{P}$-a.e. $\omega \in \Omega$.

(ii) Simple boundary conditions are sufficient for most of our purposes here. We refer to KMM11 for other useful restrictions of such types of block operators.

We write $|M|$ for the cardinality of a finite set $M$ and introduce the normalized finite-volume eigenvalue counting function

$$
\left.\left.\mathbb{N}_{\mathbb{H}_{L}}(E):=\frac{1}{2\left|\Lambda_{L}\right|} \mid \sigma\left(\mathbb{H}_{L}\right) \cap\right]-\infty, E\right] \mid=\frac{1}{2\left|\Lambda_{L}\right|} \operatorname{tr}_{\mathcal{H}_{L}^{2}}\left[1_{]-\infty, E]}\left(\mathbb{H}_{L}\right)\right],
$$

which is a non-negative random variable for every $E \in \mathbb{R}$. Here, $1_{G}$ stands for the indicator function of a set $G$ and $\operatorname{tr}_{\mathcal{H}}$ for the trace over some Hilbert space $\mathcal{H}$. The existence and self-averaging of the macroscopic limit of $\mathbb{N}_{\mathbb{H}_{L}}(E)$ is also a consequence of ergodicity.

Lemma 1.5 ([KMM11, Lemma 4.8]). There exists a (non-random) rightcontinuous probability distribution function $\mathbb{N}: \mathbb{R} \rightarrow[0,1]$, the integrated density of states of $\mathbb{H}$, and a measurable subset $\Omega_{0} \subseteq \Omega$ of full measure, $\mathbb{P}\left(\Omega_{0}\right)=1$, such that

$$
\mathbb{N}(E)=\lim _{L \rightarrow \infty} \mathbb{N}_{\mathbb{H}_{L}}^{\omega}(E)=\lim _{L \rightarrow \infty} \mathbb{E}\left[\mathbb{N}_{\mathbb{H}_{L}}(E)\right]
$$

holds for every $\omega \in \Omega_{0}$ and every continuity point $E \in \mathbb{R}$ of $\mathbb{N}$.

Since $\sigma(\mathbb{H})=\Sigma$ holds $\mathbb{P}$-a.s., one can ask for the precise location of this almost-sure spectrum. A partial answer is given by

Lemma 1.6 ([KMM11, Lemma 4.3]). Consider the random block operator $\mathbb{H}$ of Definition 1.1 . Then we have $\mathbb{P}$-a.s.

$$
\left\{ \pm \sqrt{E^{2}+\beta^{2}}: E \in \sigma(H), \beta \in \operatorname{supp}\left(\mu_{B}\right)\right\} \subseteq \sigma(\mathbb{H}) \subseteq[-r, r]
$$

where $r:=\sup _{E \in \sigma(H)}|E|+\sup _{\beta \in \operatorname{supp}\left(\mu_{B}\right)}|\beta|$.

We say that an interval $] a_{1}, a_{2}\left[\right.$, where $a_{1}, a_{2} \in \mathbb{R}$ with $a_{1}<a_{2}$, is a spectral gap of a self-adjoint operator $A$, if $] a_{1}, a_{2}\left[\cap \sigma(A)=\varnothing\right.$ and $a_{1}, a_{2} \in \sigma(A)$. In order to determine the spectral gap of $\mathbb{H}$, we will combine the above lemma with a deterministic result.

Lemma 1.7 ([KMM11, Prop. 2.10]). Consider the random block operator $\mathbb{H}$ of Definition 1.1. Then we have for $\mathbb{P}$-a.a. $\omega \in \Omega$ :

(i) If there exists $\lambda \geq 0$ such that inf $\operatorname{supp} \mu_{V} \geq \lambda$, then

$$
\left.\sigma\left(\mathbb{H}^{\omega}\right) \cap\right]-\lambda, \lambda[=\varnothing .
$$

(ii) If there exists $\beta \geq 0$ such that inf $\operatorname{supp} \mu_{B} \geq \beta$, then

$$
\left.\sigma\left(\mathbb{H}^{\omega}\right) \cap\right]-\beta, \beta[=\varnothing .
$$


(iii) If there exists $\lambda, \beta \geq 0$ such that inf $\operatorname{supp} \mu_{V} \geq \lambda$ and inf $\operatorname{supp} \mu_{B} \geq$ $\beta$, then

$$
\left.\sigma\left(\mathbb{H}^{\omega}\right) \cap\right]-\sqrt{\lambda^{2}+\beta^{2}}, \sqrt{\lambda^{2}+\beta^{2}}[=\varnothing .
$$

Remark 1.8. Lemmas 1.6 and 1.7 together provide the following two statements.

(i) If $\lambda:=\inf \operatorname{supp} \mu_{V}>0$ and $0 \in \operatorname{supp} \mu_{B}$, then $]-\lambda, \lambda[$ is $\mathbb{P}$-a.s. a spectral gap of $\mathbb{H}$ around 0 .

(ii) If $\lambda:=\inf \operatorname{supp} \mu_{V} \geq 0$ and $\beta:=\inf \operatorname{supp} \mu_{B}>0$, then ]$-\sqrt{\lambda^{2}+\beta^{2}}, \sqrt{\lambda^{2}+\beta^{2}}[$ is $\mathbb{P}$-a.s. a spectral gap of $\mathbb{H}$ around 0 .

For completeness and later use we review the main result of [KMM11, which is a Wegner estimate for the operator $\mathbb{H}$. In the next section we provide a new variant of this result. We write $\|f\|_{B V}$ for the total variation norm of some function $f: \mathbb{R} \rightarrow \mathbb{R}$.

Theorem 1.9 (Wegner estimate [KMM11, Thm. 5.1]). Consider the random block operator $\mathrm{H}$ of Definition 1.1 and assume that at least one of the following conditions is met.

(1) There exists $\lambda>0$ such that inf supp $\mu_{V} \geq \lambda$ and $\mu_{V}$ is absolutely continuous with a piecewise continuous Lebesgue density $\phi_{V}$ of bounded variation and compact support.

(2) There exists $\beta>0$ such that inf $\operatorname{supp} \mu_{B} \geq \beta$ and $\mu_{B}$ is absolutely continuous with a piecewise continuous Lebesgue density $\phi_{B}$ of bounded variation and compact support.

Then the integrated density of states $\mathbb{N}$ of $\mathbb{H}$ is Lipschitz continuous and has a bounded Lebesgue derivative, the density of states $\mathbb{D}:=\mathrm{d} \mathbb{N} / \mathrm{d} E$.

Furthermore, if hypothesis (1) holds, then we have for Lebesgue-a.a. $E \in \mathbb{R}$ that

$$
\mathrm{D}(E) \leq 2 \frac{|E|+1}{\lambda}\left\|\phi_{V}\right\|_{B V}
$$

In case of hypothesis (2), we get the estimate

$$
\mathbb{D}(E) \leq 2 \frac{|E|+1}{\beta}\left\|\phi_{B}\right\|_{B V}
$$

for Lebesgue-a.a. $E \in \mathbb{R}$.

\section{Results}

In this section we present the results of this paper. All proofs are deferred to subsequent sections. We start with a variant of Theorem 1.9 .

Theorem 2.1 (Wegner estimate). Consider the random block operator $\mathbb{H}$ of Definition 1.1 and assume that $\inf \operatorname{supp} \mu_{V} \geq 0$ and $\inf \operatorname{supp} \mu_{B} \geq 0$. Assume further that the single-site measures $\mu_{V}$ and $\mu_{B}$ are both absolutely continuous with piecewise continuous Lebesgue densities $\phi_{V}, \phi_{B}$ of bounded variation 
and compact support. Then the integrated density of states $\mathbb{N}$ is Lipschitz continuous with a bounded Lebesgue derivative $\mathbb{D}=\mathrm{d} \mathbb{N} / \mathrm{d} E$ satisfying

$$
\|\mathbb{D}\|_{\infty} \leq 2\left(\left\|\phi_{V}\right\|_{B V}+\left\|\phi_{B}\right\|_{B V}\right) .
$$

Remarks 2.2. (i) As compared to the hypotheses of the Wegner estimate from KMM11 in Theorem 1.9, the above result constitutes an improvement in that neither $H$ nor $B$ have to be bounded away from 0 . The price we have to pay is that both operators are required to be non-negative and that both probability distributions are assumed to be sufficiently regular.

(ii) As compared to the results of Theorem 1.9, we note that the present Wegner estimate is uniform in energy.

(iii) After completing this work, A. Elgart informed us that he can obtain a Wegner estimate for $\mathrm{H}$ which does not require assumptions on the supports of $\mu_{V}$ or $\mu_{B}$ E12.

Next we consider the spectral asymptotics of the integrated density of states $\mathrm{N}$ of $\mathrm{H}$ at the internal band edges.

Theorem 2.3 (Internal Lifschitz tails - upper bound). Consider the random block operator $\mathbb{H}$ of Definition 1.1. Assume that $\lambda:=\inf \operatorname{supp} \mu_{V} \geq 0$ and that the support of the measure $\mu_{V}$ consists of more than a single point. Assume further that one of the following conditions is met

(1) $\beta:=\inf \operatorname{supp} \mu_{B} \geq 0$,

(2) $\beta:=\operatorname{supsupp} \mu_{B} \leq 0$,

(3) $\quad 0 \in \operatorname{supp} \mu_{B}$, in which case we set $\beta:=0$.

Then we have

$$
\limsup _{\epsilon \searrow 0} \frac{\ln \left|\ln \left[\mathbb{N}\left(\sqrt{\lambda^{2}+\beta^{2}}+\epsilon\right)-\mathbb{N}\left(\sqrt{\lambda^{2}+\beta^{2}}\right)\right]\right|}{\ln \epsilon} \leq-\alpha
$$

with $\alpha=d / 2$ in all cases except the case $\lambda=0$ and $\beta \neq 0$, where $\alpha=d / 4$.

Remarks 2.4. (i) An analogous result holds when approaching the upper edge of the lower band $-\sqrt{\lambda^{2}+\beta^{2}}$ from below.

(ii) There is no conflict in the definition of $\beta$ in Theorem 2.3 if several of the conditions (1) - (3) hold, because this case is only possible with $\beta=0$.

(iii) If $\lambda>0$ or $\beta \neq 0$, then $\pm \sqrt{\lambda^{2}+\beta^{2}}$ are the endpoints of the spectral gap of $\mathbb{H}$; see Remark 1.8. To apply this remark in the case (2), use also unitary equivalence of $\left(\begin{array}{cc}H & B \\ B & -H\end{array}\right)$ and $\left(\begin{array}{cc}H & -B \\ -B & -H\end{array}\right)$.

(iv) Theorem 2.3 is a generalization of [KMM11, Thm. 6.1] which applies only to $\lambda>0$ and $\beta=0$.

A (mostly) complementary lower bound is provided by

Theorem 2.5 (Internal Lifschitz tails - lower bound). Consider the random block operator $\mathbb{H}$ of Definition 1.1. Assume that $\lambda:=\inf \operatorname{supp} \mu_{V} \geq 0$ and that one of the cases (1) - (3) in Theorem 2.3 applies. Assume further the 
existence of constants $C, \kappa>0$ such that for all sufficiently small $\eta>0$ the bounds

$$
\mu_{V}\left(\left[\lambda, \lambda+\eta[) \geq C \eta^{\kappa} \quad \text { and } \quad \mu_{B}(] \beta-\eta, \beta+\eta[) \geq C \eta^{\kappa}\right.\right.
$$

hold. Then we have

$$
\liminf _{\epsilon \searrow 0} \frac{\ln \left|\ln \left[\mathbb{N}\left(\sqrt{\lambda^{2}+\beta^{2}}+\epsilon\right)-\mathbb{N}\left(\sqrt{\lambda^{2}+\beta^{2}}\right)\right]\right|}{\ln \epsilon} \geq-d / 2 .
$$

Remarks 2.6. (i) Taken together, Theorems 2.3 and 2.5 imply that the random block operator $\mathbb{H}$ exhibits Lifschitz tails at the edges of its spectral gap with Lifschitz exponent $d / 2$ for all values $\lambda>0$ and $\beta \in \mathbb{R}$.

(ii) Even in the case $\lambda=\beta=0$, the block operator $\mathbb{H}$ exhibits Lifschitz tails with Lifschitz exponent $d / 2$ at energy zero. We note that there is no internal spectral edge at energy zero in this case.

(iii) In the case $\lambda=0$ and $\beta \neq 0$ we believe that the correct value of the Lifschitz exponent is $d / 2$ (rather than $d / 4$ ), as given by the lower bound in Theorem 2.5.

Finally, we turn to Anderson localization of $\mathbb{H}$ in a neighbourhood of the internal band edges. The following notion will be useful for the formulation of the result.

Definition 2.7. Given a bounded operator $\mathbb{A}$ on the Hilbert space $\mathcal{H}^{2}$ and $n, m \in \mathbb{Z}^{d}$, we introduce its $2 \times 2$-matrix-valued matrix element

$$
\mathbb{A}(n, m):=\left(\begin{array}{ll}
\left\langle\left\langle\left(\begin{array}{c}
\delta_{n} \\
0
\end{array}\right), \mathbb{A}\left(\begin{array}{c}
\delta_{m} \\
0
\end{array}\right)\right\rangle\right. & \left.\left\langle\left\langle\left(\begin{array}{c}
\delta_{n} \\
0
\end{array}\right), \mathbb{A}\left(\begin{array}{c}
0 \\
\delta_{m}
\end{array}\right)\right\rangle\right\rangle\right) \\
\left\langle\left\langle\left(\begin{array}{c}
0 \\
\delta_{n}
\end{array}\right), \mathbb{A}\left(\begin{array}{c}
\delta_{m} \\
0
\end{array}\right)\right\rangle\right. & \left\langle\left\langle\left(\begin{array}{c}
0 \\
\delta_{n}
\end{array}\right), \mathbb{A}\left(\begin{array}{c}
0 \\
\delta_{m}
\end{array}\right)\right\rangle\right)
\end{array}\right) .
$$

Here $\left\langle\langle\cdot, \cdot\rangle\right.$ stands for the canonical scalar product on the Hilbert space $\mathcal{H}^{2}$. We also fix some norm $\|\cdot\|_{2 \times 2}$ on the vector space of complex-valued $2 \times 2$ matrices.

Theorem 2.8 (Complete localization). Consider the random block operator $\mathbb{H}$ of Definition 1.1 and assume the hypotheses of Theorem 2.3. Assume further the hypotheses of Theorem 1.9 or Theorem 2.1. Then there exist constants $0<\zeta<1, C_{\zeta}>0$ and an energy interval $I:=[-a, a]$, where $a>0$, such that $I \cap \sigma(\mathbb{H}) \neq \varnothing$ holds $\mathbb{P}$-a.s. and

$$
\mathbb{E}\left(\sup _{\|f\|_{\infty} \leq 1}\left\|\left(1_{I}(\mathbb{H}) f(\mathbb{H})\right)(n, m)\right\|_{2 \times 2}\right) \leq C_{\zeta} \mathrm{e}^{-|n-m|^{\zeta}}
$$

for all $n, m \in \mathbb{Z}^{d}$. The supremum in (2.6) is taken over all Borel functions $\mathbb{R} \rightarrow \mathbb{C}$ that are pointwise bounded by 1 .

Remark 2.9. (i) The choice of the matrix norm $\|\cdot\|_{2 \times 2}$ is not crucial here. It can be replaced by any other matrix norm on the space of $2 \times 2$ matrices. 
(ii) Our proof of the theorem relies on the bootstrap multi-scale analysis of Germinet and Klein GK01. In fact, the general formulation of the bootstrap multi-scale analysis in GK01 allows an immediate and straightforward application to the present setting of random block operators. An alternative proof of localization has been carried out previously in ESS12. It adapts the fractional-moment method to rather general $k \times k$-block operators for $k \geq 2$ and applies in the strong-disorder regime. We would like to advertise the simplicity of extending the bootstrap multi-scale analysis to our block-operator setting.

(iii) Further equivalent characterizations of the region of complete localization can be found in GK04, GK06.

The RAGE Theorem leads to the following well-known corollary of Theorem 2.8 .

Corollary 2.10 (Spectral localization). Under the assumptions of Theorem 2.8 there is only pure point spectrum in $I$, that is

$$
\sigma(\mathrm{H}) \cap I=\sigma_{\mathrm{pp}}(\mathrm{H}) \cap I
$$

holds $\mathbb{P}$-a.s., and the eigenfunctions of $\mathbb{H}$ associated with eigenvalues in $I$ decay exponentially at infinity.

\section{Proof of the Wegner estimate}

The following proof of Theorem 2.1 is close to the one given in KMM11, the main difference being Lemma 3.1 below.

Proof of Theorem 2.1. In order to stress the dependence of the finite-volume operator on the families of random variables $V:=\left(V_{n}\right)_{n \in \mathbb{Z}^{d}}$ and $B:=$ $\left(B_{n}\right)_{n \in \mathbb{Z}^{d}}$, we use the notation $\mathbb{H}_{L} \equiv \mathbb{H}_{L}(V, B)$ whenever appropriate. Since

$$
\left.\mathbb{P} \text { (all eigenvalues of } \mathbb{H}_{L} \text { are non-degenerate }\right)=1 \text {, }
$$

see e.g. KS80, Prop. II.1], we infer from analytic perturbation theory that for $\mathbb{P}$-a.e. $(V, B)$ the distinct eigenvalues $E_{j} \equiv E_{j}(V, B), j=1, \ldots, 2\left|\Lambda_{L}\right|$, of $\mathrm{H}_{L}(V, B)$, which are ordered by magnitude, are all continuously differentiable (separately in each $V_{n}$ and each $B_{n}$ for $\left.n=1, \ldots,\left|\Lambda_{L}\right|\right)$ in the point $(V, B)$. For the time being we fix $E>0$ and $\epsilon>0$ with $3 \epsilon<E$. Consider a switch function $\rho \in C^{1}(\mathbb{R})$, i.e. $\rho$ is continuously differentiable, non-decreasing and obeys $0 \leq \rho \leq 1$, with $\rho(\eta)=1$ for $\eta>\epsilon$ and $\rho(\eta)=0$ for $\eta<-\epsilon$. Monotonicity gives the estimate

$$
\begin{aligned}
\operatorname{tr}_{\mathcal{H}_{L}^{2}\left[1_{[E-\epsilon, E+\epsilon[}\left(\mathbb{H}_{L}\right)\right]} & \leq \sum_{j=1}^{2\left|\Lambda_{L}\right|}\left[\rho\left(E_{j}-E+2 \epsilon\right)-\rho\left(E_{j}-E-2 \epsilon\right)\right] \\
& =\int_{E-2 \epsilon}^{E+2 \epsilon} \mathrm{d} \eta \sum_{j=1}^{2\left|\Lambda_{L}\right|} \rho^{\prime}\left(E_{j}-\eta\right) .
\end{aligned}
$$


We infer from the chain rule that

$$
\begin{aligned}
& \sum_{n \in \Lambda_{L}}\left(\frac{\partial}{\partial V_{n}}+\frac{\partial}{\partial B_{n}}\right) \rho\left(E_{j}(V, B)-\eta\right) \\
& =\rho^{\prime}\left(E_{j}(V, B)-\eta\right) \sum_{n \in \Lambda_{L}}\left(\frac{\partial}{\partial V_{n}}+\frac{\partial}{\partial B_{n}}\right) E_{j}(V, B)
\end{aligned}
$$

for all $j$, all $\eta$ and $\mathbb{P}$-a.a. $(V, B)$. Unlike the standard Anderson model, the eigenvalues $E_{j}(V, B)$ are neither monotone in the $V_{n}$ 's nor in the $B_{n}$ 's, but the choice of $\epsilon$ ensures that only positive eigenvalues contribute to the $j$-sum in (3.2). Therefore we apply Lemma 3.1 to (3.3), and estimate $\rho^{\prime}$ in (3.2) according to

$$
\rho^{\prime}\left(E_{j}(V, B)-\eta\right) \leq \sum_{n \in \Lambda_{L}}\left(\frac{\partial}{\partial V_{n}}+\frac{\partial}{\partial B_{n}}\right) \rho\left(E_{j}(V, B)-\eta\right) .
$$

Taking the expectation of (3.2) and using its product structure, we obtain

$$
\begin{aligned}
\mathbb{E}\left\{\operatorname{tr}_{\mathcal{H}_{L}^{2}}\left[1_{[E-\epsilon, E+\epsilon[}\left(\mathbb{H}_{L}\right)\right]\right\} \\
\leq \int_{E-2 \epsilon}^{E+2 \epsilon} \mathrm{d} \eta \sum_{n \in \Lambda_{L}} \int_{\mathbb{R}^{2\left|\Lambda_{L}\right|}}\left(\prod_{k \in \Lambda_{L}} \mathrm{~d} \mu_{V}\left(V_{k}\right) \mathrm{d} \mu_{B}\left(B_{k}\right)\right) \\
\quad \times \sum_{j=1}^{2\left|\Lambda_{L}\right|}\left(\frac{\partial}{\partial V_{n}}+\frac{\partial}{\partial B_{n}}\right) \rho\left(E_{j}(V, B)-\eta\right) .
\end{aligned}
$$

Each term of the $n$-sum in the previous expression can be rewritten as

$$
\begin{aligned}
\int_{\mathbb{R}^{2\left|\Lambda_{L}\right|-2}}( & \left.\prod_{k \in \Lambda_{L}: k \neq n} \mathrm{~d} \mu_{V}\left(V_{k}\right) \mathrm{d} \mu_{B}\left(B_{k}\right)\right) \\
\times & {\left[\int_{\mathbb{R}} \mathrm{d} \mu_{B}\left(B_{n}\right) \int_{\mathbb{R}} \mathrm{d} \mu_{V}\left(V_{n}\right) \sum_{j=1}^{2\left|\Lambda_{L}\right|} \frac{\partial}{\partial V_{n}} \rho\left(E_{j}(V, B)-\eta\right)\right.} \\
& \left.+\int_{\mathbb{R}} \mathrm{d} \mu_{V}\left(V_{n}\right) \int_{\mathbb{R}} \mathrm{d} \mu_{B}\left(B_{n}\right) \sum_{j=1}^{2\left|\Lambda_{L}\right|} \frac{\partial}{\partial B_{n}} \rho\left(E_{j}(V, B)-\eta\right)\right]
\end{aligned}
$$

Functions like $X_{n} \mapsto F\left(X_{n}\right):=\sum_{j=1}^{2\left|\Lambda_{L}\right|} \rho\left(E_{j}(V, B)-\eta\right)$, where $X$ stands for $V$ or $B$, are non-monotone in general. But analytic perturbation theory ensures that $F \in C^{1}(\mathbb{R})$. Moreover, $\left|F(x)-F\left(x^{\prime}\right)\right| \leq 2$ for all $x, x^{\prime} \in \mathbb{R}$ by a rank-2-perturbation argument. Therefore, Lemma 5.4. in KMM11 implies

$$
\int_{\mathbb{R}} \mathrm{d} \mu_{X}\left(X_{n}\right) \sum_{j=1}^{2\left|\Lambda_{L}\right|} \frac{\partial}{\partial X_{n}} \rho\left(E_{j}(V, B)-\eta\right) \leq 2\left\|\phi_{X}\right\|_{B V}
$$

for both $X=V$ and $X=B$. Thus, we conclude from (3.5) - (3.8) that

$$
\mathbb{E}\left\{\operatorname{tr}_{\mathcal{H}_{L}^{2}}\left[1_{[E-\epsilon, E+\epsilon[}\left(\mathbb{H}_{L}\right)\right]\right\} \leq 8 \epsilon\left|\Lambda_{L}\right|\left(\left\|\phi_{V}\right\|_{B V}+\left\|\phi_{B}\right\|_{B V}\right)
$$


for every $E>0$ and every $0<\epsilon<E / 3$. This bound and dominated convergence establish Lipschitz continuity of the integrated density of states $\mathbb{N}$ on $\mathbb{R}_{>0}$ with Lipschitz constant $2\left(\left\|\phi_{V}\right\|_{B V}+\left\|\phi_{B}\right\|_{B V}\right)$. But due to the symmetry of the spectrum, see Remark 1.2 (ii), this extends to $\mathbb{R} \backslash\{0\}$. Furthermore, since $\mathbb{N}$ is a continuous function on the whole real line $\mathbb{R}$ - which follows from standard arguments as in [K08, Thm. 5.14] - this yields Lipschitz continuity on $\mathbb{R}$ with the same constant.

One of the main estimates in the previous proof is provided by the following deterministic result.

Lemma 3.1. Assume that $H_{L} \geq 0, B_{L} \geq 0$ and let $E(V, B)>0$ be a simple eigenvalue of $\mathrm{H}_{L}(V, B)$. Then we have

$$
\sum_{n \in \Lambda_{L}}\left(\frac{\partial}{\partial V_{n}}+\frac{\partial}{\partial B_{n}}\right) E(V, B) \geq 1 .
$$

Proof. Let $\Psi=\left(\psi_{1}, \psi_{2}\right)$ be a normalized eigenvector corresponding to the eigenvalue $E \equiv E(V, B)$ of the operator $\mathbb{H}_{L} \equiv \mathbb{H}_{L}(V, B)$, i.e. $\left\langle\psi_{1}, \psi_{1}\right\rangle+$ $\left\langle\psi_{2}, \psi_{2}\right\rangle=1$ and

$$
\begin{aligned}
& H_{L} \psi_{1}+B \psi_{2}=E \psi_{1}, \\
& B \psi_{1}-H_{L} \psi_{2}=E \psi_{2} .
\end{aligned}
$$

The Feynman-Hellmann formula for a non-degenerate eigenvalue and (3.11) imply

$$
\begin{aligned}
E \sum_{j \in \Lambda_{L}}( & \left.\frac{\partial}{\partial V_{j}}+\frac{\partial}{\partial B_{j}}\right) E \\
= & E\left(\left\langle\psi_{1}, \psi_{1}\right\rangle-\left\langle\psi_{2}, \psi_{2}\right\rangle+\left\langle\psi_{1}, \psi_{2}\right\rangle+\left\langle\psi_{2}, \psi_{1}\right\rangle\right) \\
= & \left\langle\psi_{1}, H_{L} \psi_{1}+B \psi_{2}\right\rangle-\left\langle B \psi_{1}-H_{L} \psi_{2}, \psi_{2}\right\rangle+\left\langle\psi_{1}, B \psi_{1}-H_{L} \psi_{2}\right\rangle \\
& +\left\langle\psi_{2}, H_{L} \psi_{1}+B \psi_{2}\right\rangle \\
= & \left\langle\psi_{1}, H_{L} \psi_{1}\right\rangle+\left\langle\psi_{2}, H_{L} \psi_{2}\right\rangle+\left\langle\psi_{1}, B \psi_{1}\right\rangle+\left\langle\psi_{2}, B \psi_{2}\right\rangle .
\end{aligned}
$$

In the last step we used that the operator $\mathbb{H}_{L}$ is a real symmetric matrix and, therefore, the eigenvector $\Psi$ can be chosen to be real. Since $B \geq 0$, we have

$$
\left\langle\psi_{1}, B \psi_{1}\right\rangle+\left\langle\psi_{2}, B \psi_{2}\right\rangle \geq\left\langle\psi_{1}, B \psi_{2}\right\rangle+\left\langle\psi_{2}, B \psi_{1}\right\rangle
$$

This and $H_{L} \geq 0$ yield the lower bound

$$
\left\langle\psi_{1}, H_{L} \psi_{1}+B \psi_{2}\right\rangle+\left\langle\psi_{2}, B \psi_{1}-H_{L} \psi_{2}\right\rangle=E\left(\left\langle\psi_{1}, \psi_{1}\right\rangle+\left\langle\psi_{2}, \psi_{2}\right\rangle\right)=E
$$

for the r.h.s. of (3.12).

\section{Proof of Lifschitz tails}

In this section we prove Theorems 2.3 and 2.5 . 
The idea behind the proof of Theorem 2.3 is to estimate the integrated density of states of $\mathbb{H}$ in terms of the integrated density of states of the operator

$$
\mathrm{H}(\beta):=\left(\begin{array}{cc}
H & \beta \mathbf{1} \\
\beta \mathbf{1} & -H
\end{array}\right),
$$

on $\mathcal{H}^{2}$, where $\beta$ is as in Theorem 2.3 and $\mathbf{1}$ denotes the unit operator on $\ell^{2}\left(\mathbb{Z}^{d}\right)$. This is useful because we explicitly know the relation between the spectra of $\mathbb{H}(\beta)$ and $H$, and because the discrete Schrödinger operator $H$ of the Anderson model exhibits Lifschitz tails at the edges of its spectrum. For the lower spectral edge of $H$ the upper Lifschitz-tail estimate is summarized in the next lemma, for a proof see e.g. [CL90, PF92, K89].

Lemma 4.1 (Upper Lifschitz-tail estimate for $\boldsymbol{H}$ ). Let $H$ be the discrete random Schrödinger operator of the Anderson model as in Definition 1.1. Assume in addition that the single-site probability measure $\mu_{V}$ is not concentrated in a single point. Then, the integrated density of states $N_{H}$ of the operator $H$ obeys

$$
\limsup _{\epsilon \searrow 0} \frac{\ln \left|\ln \left[N_{H}(\lambda+\epsilon)\right]\right|}{\ln \epsilon} \leq-\frac{d}{2},
$$

where $\lambda:=\inf \operatorname{supp} \mu_{V}=\inf \sigma(H)$ is the infimum of the almost-sure spectrum of $H$.

The remaining arguments needed for the proof of Theorem 2.3 are all deterministic. The next lemma, which is a particular case of [T08, Thm. 1.9.1], provides a variational principle for the positive spectrum of the finite-volume block operator $\mathbb{H}_{L}$.

Lemma 4.2 (Min-max-max principle). Given $A, B$ and $D$ self adjoint operators on $\mathcal{H}=l^{2}\left(\Lambda_{L}\right)$ with $A>-D$, define the block operator $\mathbb{A}:=\left(\begin{array}{cc}A & B \\ B & -D\end{array}\right)$ on $\mathcal{H}^{2}$. Then

(i) there are precisely $\left|\Lambda_{L}\right|$ eigenvalues of $\mathbb{A}, \lambda_{1}, \ldots, \lambda_{\left|\Lambda_{L}\right|}$, with $\lambda_{j}>$ $\sup \sigma(-D)$ and

(ii) the eigenvalues $\lambda_{j}>\sup \sigma(-D), j=1, \ldots,\left|\Lambda_{L}\right|$, ordered by magnitude and repeated according to their multiplicity, are given by

$$
\begin{aligned}
\lambda_{j}=\min _{\substack{\mathcal{V} \subset \ell^{2}\left(\Lambda_{L}\right): \\
\operatorname{dim} \mathcal{V}=j}} \max _{\substack{f \in \mathcal{V}: \\
\|f\|=1}} \max _{\substack{g \in \ell^{2}\left(\Lambda_{L}\right): \\
\|g\|=1}}\left\{\frac{\langle f, A f\rangle-\langle g, D g\rangle}{2}\right. \\
\left.+\sqrt{\left(\frac{\langle f, A f\rangle+\langle g, D g\rangle}{2}\right)^{2}+|\langle f, B g\rangle|^{2}}\right\} .
\end{aligned}
$$

This variational characterization will serve to relate the positive spectrum of $\mathbb{H}_{L}$ to that of $\mathbb{H}_{L}(\beta)$, which is the restriction of $\mathbb{H}(\beta)$ to $\mathcal{H}_{L}^{2}$ in analogy with Definition 1.3. Finally, we relate the spectrum of $\mathrm{H}_{L}(\beta)$ to that of its diagonal block $H_{L}$. 
Lemma 4.3 ([KMM11, Prop. 3.1]). The spectrum of $\mathrm{H}_{L}(\beta)$ is given by

$$
\sigma\left(\mathbb{H}_{L}(\beta)\right)=\left\{ \pm \sqrt{E^{2}+\beta^{2}}: E \in \sigma\left(H_{L}\right)\right\} .
$$

Now we are prepared for the

Proof of Theorem 2.3. Since $H \geq 0$ we have $H_{L}>0$ and can apply Lemma 4.2. Setting $f=g$ there and noting that $\mathbb{P}$-a.s. $\beta=\inf \sigma(|B|)$, we infer

$$
\begin{aligned}
\lambda_{j} \geq \min _{\substack{\mathcal{V} \subset \ell^{2}\left(\Lambda_{L}\right): \\
\operatorname{dim} \mathcal{V}=j}} \max _{\substack{f \in \mathcal{V}: \\
\|f\|=1}} \sqrt{\left\langle f, H_{L} f\right\rangle^{2}+\langle f, B f\rangle^{2}} \\
\geq \min _{\substack{\mathcal{V} \subset \ell^{2}\left(\Lambda_{L}\right): \\
\operatorname{dim} \mathcal{V}=j}} \max _{\substack{f \in \mathcal{V}: \\
\|f\|=1}} \sqrt{\left\langle f, H_{L} f\right\rangle^{2}+\beta^{2}} \\
=\left[\left(\min _{\substack{\mathcal{V} \subset \ell^{2}\left(\Lambda_{L}\right): \\
\operatorname{dim} \mathcal{V}=j}} \max _{\substack{f \in \mathcal{V}: \\
\|f\|=1}}\left\langle f, H_{L} f\right\rangle\right)^{2}+\beta^{2}\right]^{1 / 2}
\end{aligned}
$$

for every $j=1, \ldots,\left|\Lambda_{L}\right|$. We denote the positive eigenvalues of $\mathrm{H}_{L}(\beta)$ by $0<\mu_{1} \leq \ldots \leq \mu_{\left|\Lambda_{L}\right|}$. The min-max principle for $H_{L}$ and Lemma 4.3 then imply

$$
\lambda_{j} \geq \mu_{j}
$$

for every $j=1, \ldots,\left|\Lambda_{L}\right|$. Symmetry of the spectra of $\mathbb{H}_{L}$ and $\mathbb{H}_{L}(\beta)$, see Remark 1.2 (ii) the strict positivity $H_{L}>\inf \sigma(H)=\lambda \geq 0$ and Lemma 1.7 (iii) imply

$$
\mathbb{N}_{\mathbb{H}_{L}}\left(\sqrt{\lambda^{2}+\beta^{2}}\right)=\mathbb{N}_{\mathbb{H}_{L}(\beta)}\left(\sqrt{\lambda^{2}+\beta^{2}}\right)=\frac{1}{2} .
$$

Setting $E:=\sqrt{\lambda^{2}+\beta^{2}}+\epsilon$ for $\epsilon>0$, Eqs. (4.7) and (4.6) give the estimate

$$
\begin{aligned}
\mathbb{N}_{\mathbb{H}_{L}}(E)-\mathbb{N}_{\mathbb{H}_{L}} & \left(\sqrt{\lambda^{2}+\beta^{2}}\right) \\
& \leq \mathbb{N}_{\mathbb{H}_{L}(\beta)}(E)-\mathbb{N}_{\mathbb{H}_{L}(\beta)}\left(\sqrt{\lambda^{2}+\beta^{2}}\right) \\
& =\frac{1}{2\left|\Lambda_{L}\right|} \mid\left\{\mu \in \sigma\left(\mathbb{H}_{L}(\beta)\right): \mu \in\left[\sqrt{\lambda^{2}+\beta^{2}}, E[\} \mid\right.\right. \\
& =\frac{1}{2\left|\Lambda_{L}\right|} \mid\left\{\widetilde{\mu} \in \sigma\left(H_{L}\right): \widetilde{\mu} \in\left[\lambda, \sqrt{E^{2}-\beta^{2}}[\} \mid\right.\right. \\
& =\frac{1}{2} N_{H_{L}}\left(\sqrt{E^{2}-\beta^{2}}\right),
\end{aligned}
$$


where we have used Lemma 4.3 for the second equality. Therefore we get in the limit $L \rightarrow \infty$ and using Lemma 4.1

$$
\begin{aligned}
\limsup _{\epsilon \searrow 0} \frac{\ln \left|\ln \left[\mathbb{N}\left(\sqrt{\lambda^{2}+\beta^{2}}+\epsilon\right)-\mathbb{N}\left(\sqrt{\lambda^{2}+\beta^{2}}\right)\right]\right|}{\ln \epsilon} \\
\quad \leq \limsup _{\epsilon \searrow 0} \frac{\ln \left|\ln N_{H}\left(\left[\left(\sqrt{\lambda^{2}+\beta^{2}}+\epsilon\right)^{2}-\beta^{2}\right]^{1 / 2}\right)\right|}{\ln \epsilon} \\
\quad=\limsup _{\widetilde{\epsilon} \sup _{0} \frac{\ln \left|\ln N_{H}(\lambda+\widetilde{\epsilon})\right|}{\xi \ln \widetilde{\epsilon}}} \\
\leq-\frac{d}{2 \xi}
\end{aligned}
$$

with $\xi=1$ in all cases except the case of $\lambda=0$ and $\beta \neq 0$, where $\xi=2$.

In the remaining part of this section we turn to the lower bound for Lifschitz tails.

Proof of Theorem 2.5. We use Dirichlet-Neumann bracketing. Therefore we define, following KMM11, Def. 4.6], the Dirichlet-bracketing restriction of the block operator as

$$
\mathbb{H}_{L}^{+}:=\left(\begin{array}{cc}
H_{L}^{D} & B \\
B & -H_{L}^{N}
\end{array}\right)
$$

where $H_{L}^{N}$ and $H_{L}^{D}$ denote the restriction of $H$ to the cube $\Lambda_{L}, L \in \mathbb{N}$, with Neumann, respectively Dirichlet boundary conditions on the Laplacian; for a precise definition see [K08, Sect. 5.2]. Setting $\widetilde{H}_{L}^{D}:=H_{L}^{D}-\lambda \mathbf{1}, \widetilde{B}:=$ $B-\beta \mathbf{1}$ and using Lemma 4.2, we obtain for the $j$-th positive eigenvalue, $j=1, \ldots,\left|\Lambda_{L}\right|$,

$$
\begin{aligned}
\lambda_{j}\left(\mathbb{H}_{L}^{D}\right)= & \min _{\substack{\mathcal{V} \subset \ell^{2}\left(\Lambda_{L}\right): \\
\operatorname{dim} \mathcal{V}=j}} \max _{\substack{f \in \mathcal{V}: \\
\|f\|=1}} \max _{\substack{g \in \ell^{2}\left(\Lambda_{L}\right): \\
\|g\|=1}}\left\{\frac{\left\langle f, \widetilde{H}_{L}^{D} f\right\rangle-\left\langle g, \widetilde{H}_{L}^{N} g\right\rangle}{2}\right. \\
& \left.+\sqrt{\left(\lambda+\frac{\left\langle f, \widetilde{H}_{L}^{D} f\right\rangle+\left\langle g, \widetilde{H}_{L}^{N} g\right\rangle}{2}\right)^{2}+|\beta+\langle f, \widetilde{B} g\rangle|^{2}}\right\} .
\end{aligned}
$$

The elementary inequality

$$
\sqrt{(\lambda+a)^{2}+(\beta+b)^{2}} \leq \sqrt{\lambda^{2}+\beta^{2}}+a+b
$$

holds for every $a, b \geq 0$ and $\lambda, \beta \in \mathbb{R}$.

Together with the estimate $|\beta+\langle f, \widetilde{B} g\rangle| \leq|\beta|+\left\langle f, \widetilde{B}^{2} f\right\rangle^{1 / 2}$, this yields

$$
\lambda_{j}\left(\mathbb{H}_{L}^{D}\right) \leq \sqrt{\lambda^{2}+\beta^{2}}+\min _{\substack{\mathcal{V} \subset \ell^{2}\left(\Lambda_{L}\right): \\ \operatorname{dim} \mathcal{V}=j}} \max _{\substack{f \in \mathcal{V}: \\\|f\|=1}}\left\{\left\langle f, \widetilde{H}_{L}^{D} f\right\rangle+\left\langle f, \widetilde{B}^{2} f\right\rangle^{1 / 2}\right\} .
$$

On the other hand, 4.11) implies

$$
\lambda_{j}\left(\mathbb{H}_{L}^{D}\right)>\sqrt{\lambda^{2}+\beta^{2}}
$$


for every $j=1, \ldots,\left|\Lambda_{L}\right|$. From this and Lemma 4.2 we conclude that $\mathbb{E}\left[\mathbb{N}_{\mathbb{H}_{L}^{+}}\left(\sqrt{\lambda^{2}+\beta^{2}}\right)\right]=1 / 2$. Similarly, using the symmetry of the spectrum and continuity of the integrated density of states (cf. the proof of $\mathrm{K} 08$, Lemma 5.13]), we obtain $\mathbb{E}\left[\mathbb{N}\left(\sqrt{\lambda^{2}+\beta^{2}}\right)\right]=1 / 2$. These two equalities and the estimate $\mathbb{N}(E) \geq \mathbb{E}\left[\mathbb{N}_{\mathbb{H}_{L}^{+}}(E)\right]$ for every $E \in \mathbb{R}$ [KMM11, Lemma 4.8(ii)] yield

$$
\begin{aligned}
\mathbb{N}\left(\sqrt{\lambda^{2}+\beta^{2}}\right. & +\epsilon)-\mathbb{N}\left(\sqrt{\lambda^{2}+\beta^{2}}\right) \\
& \geq \mathbb{E}\left[\mathbb{N}_{\mathbb{H}_{L}^{+}}\left(\sqrt{\lambda^{2}+\beta^{2}}+\epsilon\right)\right]-\mathbb{E}\left[\mathbb{N}_{\mathbb{H}_{L}^{+}}\left(\sqrt{\lambda^{2}+\beta^{2}}\right)\right] \\
& \geq \frac{1}{2\left|\Lambda_{L}\right|} \mathbb{P}\left(\lambda _ { 1 } ( \mathbb { H } _ { L } ^ { D } ) \in \left[\sqrt{\lambda^{2}+\beta^{2}}, \sqrt{\lambda^{2}+\beta^{2}}+\epsilon[)\right.\right. \\
& \geq \frac{1}{2\left|\Lambda_{L}\right|} \mathbb{P}\left(\left\langle\psi, \widetilde{H}_{L}^{D} \psi\right\rangle+\left\langle\psi, \widetilde{B}^{2} \psi\right\rangle^{1 / 2}<\epsilon\right)
\end{aligned}
$$

for every $L \in \mathbb{N}, \epsilon>0$ and every normalized test function $\psi \in l^{2}\left(\Lambda_{L}\right)$.

Following [K08, Sect. 6.3], we choose $\psi:=\frac{1}{\left\|\psi_{1}\right\|} \psi_{1}(n)$, where $\psi_{1}(n):=\frac{L}{2}-$ $|n|_{\infty}$ for $n \in \Lambda_{L}$. This implies $\left\langle\psi, H_{0, L}^{D} \psi\right\rangle \leq c_{0} L^{-2}$ with some constant $c_{0}>0$. Next we choose $L$ to be the smallest integer such that

$$
c_{0} L^{-2}<\epsilon / 2
$$

and estimate

$$
\begin{aligned}
\mathbb{P}\left(\left\langle\psi, \widetilde{H}_{L}^{D} \psi\right\rangle\right. & \left.+\left\langle\psi, \widetilde{B}^{2} \psi\right\rangle^{1 / 2}<\epsilon\right) \\
& \geq \mathbb{P}\left(\langle\psi,(V-\lambda \mathbf{1}) \psi\rangle+\left\langle\psi,(B-\beta \mathbf{1})^{2} \psi\right\rangle^{1 / 2}<\epsilon / 2\right) \\
& \geq \mathbb{P}\left(\forall n \in \Lambda_{L}: V(n)-\lambda<\epsilon / 4 \text { and }|B(n)-\beta|<\epsilon / 4\right) \\
& =\left\{\mu _ { V } \left([ \lambda , \lambda + \epsilon / 4 [ ) \} ^ { | \Lambda _ { L } | } \left\{\mu _ { B } \left([\beta-\epsilon / 4, \beta+\epsilon / 4[)\}^{\left|\Lambda_{L}\right|} .\right.\right.\right.\right.
\end{aligned}
$$

The theorem now follows with (4.15) and the assumption (2.3).

\section{Proof of localization}

Our proof relies on the bootstrap multi-scale analysis introduced in GK01, which yields complete localization in a rather general setting. Apart from one natural adaptation for multiplication operators - see below - we are only left to check whether the assumptions on the random operator are fulfilled by our model. We start with some notions.

Definition 5.1. We introduce the boundary of a cube $\Lambda \subset \mathbb{Z}^{d}$ by $\partial \Lambda:=\left\{(n, m) \in \mathbb{Z}^{d} \times \mathbb{Z}^{d}:|n-m|=1, n \in \Lambda, m \notin \Lambda\right.$ or $\left.n \notin \Lambda, m \in \Lambda\right\}$, its inner boundary by

$$
\partial^{i} \Lambda:=\{n \in \Lambda: \exists m \notin \Lambda \text { such that }|n-m|=1\}
$$


and its outer boundary by

$$
\partial^{o} \Lambda:=\{n \notin \Lambda: \exists m \in \Lambda \text { such that }|n-m|=1\} .
$$

We write $\Lambda_{1} \sqsubset \Lambda_{2}$ if $\partial \Lambda_{1} \subset \Lambda_{2} \times \Lambda_{2}$. Furthermore for $\Lambda_{1} \sqsubset \Lambda_{2} \subseteq \mathbb{Z}^{d}$ we define the boundary operator $\Gamma_{\Lambda_{1}}^{\Lambda_{2}} \equiv \Gamma_{\Lambda_{1}}$ on $\ell^{2}\left(\Lambda_{2}\right)$ in terms of its matrix elements

$$
\left\langle\delta_{n}, \Gamma_{\Lambda_{1}} \delta_{m}\right\rangle:=\left\{\begin{array}{lll}
-1, & & (n, m) \in \partial \Lambda_{1}, \\
0, & & (n, m) \in\left(\Lambda_{2} \times \Lambda_{2}\right) \backslash \partial \Lambda_{1} .
\end{array}\right.
$$

We lift $\Gamma_{\Lambda_{1}}$ to a bounded operator on $\ell^{2}\left(\Lambda_{2}\right) \oplus \ell^{2}\left(\Lambda_{2}\right)$ by setting

$$
\mathbb{\Gamma}_{\Lambda_{1}}:=\Gamma_{\Lambda_{1}} \oplus\left(-\Gamma_{\Lambda_{1}}\right)
$$

In contrast, given subsets $\Lambda \subset \Lambda^{\prime} \subseteq \mathbb{Z}^{d}$, we lift the multiplication operator $1_{\Lambda}$ on $\ell^{2}\left(\Lambda^{\prime}\right)$, corresponding to the indicator function of $\Lambda$, to the sum space $\ell^{2}\left(\Lambda^{\prime}\right) \oplus \ell^{2}\left(\Lambda^{\prime}\right)$ by setting

$$
\mathbb{1}_{\Lambda}:=1_{\Lambda} \oplus 1_{\Lambda}
$$

In slight abuse of notation we also write $\mathbb{1}_{n}:=\mathbb{1}_{\{n\}}$ for $n \in \mathbb{Z}^{d}$. Finally, given an energy $E \notin \sigma\left(\mathbb{H}_{\Lambda}\right)$, we use the abbreviation $\mathbb{G}_{\Lambda}(E):=\left(\mathbb{H}_{\Lambda}-E\right)^{-1}$ for the resolvent of $\mathrm{H}_{\Lambda}$.

Proof of Theorem 2.8. We apply [GK01, Thm. 3.8] on the Hilbert space $\mathcal{H}^{2}$, with the random operator $\mathbb{H}$ and with $\mathbb{1}_{\Lambda}$ playing the role of the multiplication operator $\chi_{\Lambda}$ in GK01. The deterministic Assumptions SLI and EDI will be checked in Lemmas 5.2 and Lemma 5.3 below. We note a slight structural difference between the statement of Lemma 5.3 and the EDI-property in GK01: the factor $\left\|\mathbb{1}_{\partial^{\circ} \Lambda} \Psi\right\|$ in (5.12) evaluates $\Psi$ outside the cube $\Lambda$. However, this factor plays only a role in the proof of Lemma 4.1 in GK01, and Eq. (4.3) - (4.4) in that proof show that this difference is irrelevant.

The next important hypothesis of Thm. 3.8 in GK01 is the Wegner Assumption $W$, which follows from Theorem 1.9 or 2.1 for our model with $b=1$ (more precisely from the finite-volume estimates - e.g. (3.9) - in the proofs of those theorems). The remaining assumptions $I A D, N E$ and $S G E E$ are obviously correct because we work with a discrete model with i.i.d. random coupling constants. Finally, the initial-scale estimate follows from Theorem 5.5 below, see also Remark 3.7 in GK01.

Having collected all the aforementioned properties, Cor. 3.12 of GK01 implies that the claim of Thm. 3.8 holds for all energies in some interval $I:=[-a, a]$, where $a>\sqrt{\lambda^{2}+\beta^{2}}$ so that $I$ overlaps with the almost-sure spectrum of $\mathbb{H}$ according to Lemma 1.6. The claim of Thm. 3.8 then reads

$$
\mathbb{E}\left(\sup _{\|f\|_{\infty} \leq 1}\left\|\mathbb{1}_{n} 1_{I}(\mathbb{H}) f(\mathbb{H}) \mathbb{1}_{m}\right\|_{H S}^{2}\right) \leq C_{\zeta} \mathrm{e}^{-|n-m|^{\zeta}}
$$

for all $n, m \in \mathbb{Z}^{d}$. Here, $\|\mathbb{A}\|_{H S}$ is the Hilbert-Schmidt norm of an operator $\mathbb{A}$ on $\mathcal{H}^{2}$. To get to our formulation in (2.6) we remark that

$$
\left\|\mathbb{1}_{n} \mathbb{A} \mathbb{1}_{m}\right\|_{H S}=\|\mathbb{A}(n, m)\|_{2 \times 2},
$$


where, on the right-hand side, we use the notation introduced in (2.5), and $\|\cdot\|_{2 \times 2}$ stands for the Hilbert-Schmidt norm of a $2 \times 2$-matrix. Replacing the latter by any other norm on the $2 \times 2$-matrices as in (2.6), merely requires a possible adjustment of the constant $C_{\zeta}$.

Next we deal with the deterministic assumptions required by the bootstrap multi-scale analysis. The first one is a consequence of the geometric resolvent equation (5.11).

Lemma 5.2 (SLI). Let $\Lambda_{1} \sqsubset \Lambda_{2} \sqsubset \Lambda_{3}$. Then we have for $E \notin\left(\sigma\left(\mathbb{H}_{\Lambda_{2}}\right) \cup\right.$ $\left.\sigma\left(\mathrm{H}_{\Lambda_{3}}\right)\right)$ the inequality

$$
\left\|\mathbb{1}_{\partial^{i} \Lambda_{3}} \mathbb{G}_{\Lambda_{3}}(E) \mathbb{1}_{\Lambda_{1}}\right\| \leq \gamma\left\|\mathbb{1}_{\partial^{i} \Lambda_{3}} \mathbb{G}_{\Lambda_{3}}(E) \mathbb{1}_{\partial^{\circ} \Lambda_{2}}\right\|\left\|\mathbb{1}_{\partial^{i} \Lambda_{2}} \mathbb{G}_{\Lambda_{2}}(E) \mathbb{1}_{\Lambda_{1}}\right\|,
$$

where $\gamma>0$ depends only on the space dimension $d$ and the norm is the operator norm.

Proof. The identity

$$
\mathbb{H}_{\Lambda_{3}}=\left(\mathbb{H}_{\Lambda_{2}} \oplus \mathbb{H}_{\Lambda_{3} \backslash \Lambda_{2}}\right)+\mathbb{\Gamma}_{\Lambda_{2}}
$$

and the resolvent equation imply

$$
\begin{aligned}
\mathbb{1}_{\partial^{i} \Lambda_{3}} \mathbb{G}_{\Lambda_{3}}(E) \mathbb{1}_{\Lambda_{1}} & =-\mathbb{1}_{\partial^{i} \Lambda_{3}} \mathbb{G}_{\Lambda_{3}}(E) \mathbb{\Gamma}_{\Lambda_{2}} \mathbb{G}_{\Lambda_{2}}(E) \mathbb{1}_{\Lambda_{1}} \\
& =-\mathbb{1}_{\partial^{i} \Lambda_{3}} \mathbb{G}_{\Lambda_{3}}(E) \mathbb{1}_{\partial^{\circ} \Lambda_{2}} \mathbb{\Gamma}_{\Lambda_{2}} \mathbb{1}_{\partial^{i} \Lambda_{2}} \mathbb{G}_{\Lambda_{2}}(E) \mathbb{1}_{\Lambda_{1}},
\end{aligned}
$$

where we used that $\mathbb{\Gamma}_{\Lambda_{2}} \mathbb{1}_{\Lambda_{2}}=\mathbb{1}_{\partial^{\circ} \Lambda_{2}} \mathbb{\Gamma}_{\Lambda_{2}} \mathbb{1}_{\partial^{i} \Lambda_{2}}$. Taking the norm and observing that $\gamma:=\left\|\mathbb{\Gamma}_{\Lambda_{2}}\right\|$ depends only on the space dimension $d$, yields the statement.

A similar argument proves

Lemma 5.3 (EDI). Let $\Psi$ be a generalized eigenfunction of $\mathbb{H}$ with generalized eigenvalue $E$ and let $\gamma$ be the constant from the previous lemma. Then we have for any $\Lambda$ such that $E \notin \sigma\left(\mathbb{H}_{\Lambda}\right)$ and $n \in \Lambda$

$$
\left\|\mathbb{1}_{n} \Psi\right\| \leq \gamma\left\|\mathbb{1}_{n} \mathbb{G}_{\Lambda}(E) \mathbb{1}_{\partial^{i} \Lambda}\right\|\left\|\mathbb{1}_{\partial^{\circ} \Lambda} \Psi\right\| .
$$

Proof. We infer from (5.10) with $\Lambda_{3}=\mathbb{Z}^{d}$ and $\Lambda_{2}=\Lambda$ that

$$
\left(\mathbb{H}_{\Lambda} \oplus \mathbb{H}_{\mathbb{Z}^{d} \backslash \Lambda}-E\right) \Psi=-\mathbb{\Gamma}_{\Lambda} \Psi .
$$

Since $E \notin \sigma\left(\mathbb{H}_{\Lambda}\right)$ and $n \in \Lambda$, this implies $\mathbb{1}_{n} \Psi=-\mathbb{1}_{n} \mathbb{G}_{\Lambda}(E) \mathbb{\Gamma}_{\Lambda} \Psi$. The identity $\mathbb{1}_{\Lambda} \mathbb{\Gamma}_{\Lambda}=\mathbb{1}_{\partial^{i} \Lambda} \mathbb{\Gamma}_{\Lambda} \mathbb{1}_{\partial^{\circ} \Lambda}$ and taking norms finishes the proof.

The remaining part of this section is concerned with the verification of the initial-scale estimate.

Definition 5.4. Let $\theta>0$ and $E \in \mathbb{R}$. A cube $\Lambda_{L} \subset \mathbb{Z}^{d}, L \in 6 \mathbb{N}$, is $(\theta, E)$ suitable, if $E \notin \sigma\left(\mathrm{H}_{L}\right)$ and

$$
\left\|\mathbb{1}_{\partial^{i} \Lambda_{L}} \mathbb{G}_{\Lambda_{L}}(E) \mathbb{1}_{\Lambda_{L / 3}}\right\|<L^{-\theta} .
$$


Theorem 5.5 (Initial estimate). Consider the random block operator $\mathbb{H}$ of Definition 1.1 and assume the hypotheses of Theorem 2.3. Then there exist constants $\theta>d$ and $p>0$ such that for every length $L \in 6 \mathbb{N}$ sufficiently large the following holds: there exists an energy $a_{L}>\sqrt{\lambda^{2}+\beta^{2}}$ such that

$$
\mathbb{P}\left(\Lambda_{L} \text { is }(\theta, E) \text {-suitable }\right)>1-L^{-p}
$$

for every energy $E \in\left[-a_{L}, a_{L}\right]$.

We use Lifschitz tails at the internal bad edges to prove Theorem 5.5. Lifschitz tails arise from a small probability for finding an eigenvalue close to the spectral edge. This mechanism also yields the high probability for the event in (5.15). As in the proof of Lifschitz tails for $\mathrm{H}$ in Sect. 4, we will reduce this to a corresponding statement for $H$.

Lemma 5.6 (Lifschitz-tail estimate [K08, Eq. (11.23)]). Let $H$ be the discrete random Schrödinger operator of the Anderson model as in Definition 1.1. Assume in addition that the single-site probability measure $\mu_{V}$ is not concentrated in a single point and let $\lambda:=\inf \operatorname{supp} \mu_{V}$ be the infimum of the almost-sure spectrum of $H$. Then, given any $C, p>0$, we have for every $L \in \mathbb{N}$ sufficiently large

$$
\mathbb{P}\left(\inf \sigma\left(H_{L}\right) \leq \lambda+C L^{-1 / 2}\right) \leq \frac{1}{L^{p}} .
$$

As a second ingredient for the initial-scale estimate we need some natural decay of the Green function of $\mathbb{H}_{L}$.

Lemma 5.7 (Combes-Thomas estimate). For $L \in \mathbb{N}$ consider the finite-volume block operator $\mathbb{H}_{L}$ of Definition 1.3 . Fix $E \in \mathbb{R}$ with $\operatorname{dist}\left(E, \sigma\left(\mathbb{H}_{L}\right)\right) \geq \delta$ for some $\delta \leq 1$. Then we have for all $n, m \in \mathbb{Z}^{d}$ that

$$
\left\|\mathbb{1}_{n} \mathbb{G}_{\Lambda_{L}}(E) \mathbb{1}_{m}\right\| \leq \frac{4}{\delta} \mathrm{e}^{-(\delta / 12 d)|n-m|} .
$$

Proof. We have patterned the lemma after [K08, Thm. 11.2], and its proof follows from a straightforward adaptation to random block operators of the proof there. Details can be found in G11.

We are now ready for the

Proof of Theorem 5.5. Fix $\theta>d$, let $L \in 6 \mathbb{N}$, set $a_{L}:=\sqrt{\lambda^{2}+\beta^{2}}+L^{-1 / 2}$ and pick any $E \in\left[-a_{L}, a_{L}\right]$. Assuming that the event

$$
\inf \sigma\left(\left|\mathbb{H}_{L}\right|\right)>a_{L}+L^{-1 / 2}
$$

holds, then the Combes-Thomas estimate yields

$$
\left\|\mathbb{1}_{n} \mathbb{G}_{\Lambda_{L}}(E) \mathbb{1}_{m}\right\| \leq 4 \sqrt{L} \mathrm{e}^{-|n-m| /(12 d \sqrt{L})} \leq 4 \sqrt{L} \mathrm{e}^{-\sqrt{L} /(48 d)}
$$

for all $n \in \partial^{i} \Lambda_{L}$ and all $m \in \Lambda_{L / 3}$. Thus, provided $L$ is sufficiently large, the event (5.18) implies that the cube $\Lambda_{L}$ is $(\theta, E)$-suitable. Negating this implication, we conclude

$$
\mathbb{P}\left(\Lambda_{L} \text { is not }(\theta, E) \text {-suitable }\right) \leq \mathbb{P}\left(\inf \sigma\left(\left|\mathbb{H}_{L}\right|\right) \leq a_{L}+L^{-1 / 2}\right) .
$$


The symmetry of the spectrum and the ordering (4.6) of the eigenvalues of the operators $\mathrm{H}_{L}$ and $\mathrm{H}_{L}(\beta)$ gives

$$
\begin{aligned}
\mathbb{P}\left(\Lambda_{L} \text { is not }(\theta, E) \text {-suitable }\right) & \leq \mathbb{P}\left(\inf \sigma\left(\left|\mathbb{H}_{L}(\beta)\right|\right) \leq a_{L}+L^{-1 / 2}\right) \\
& \leq \mathbb{P}\left(\inf \sigma\left(\left|\mathbb{H}_{L}(\beta)\right|\right) \leq \sqrt{\left(\lambda+C L^{-1 / 2}\right)^{2}+\beta^{2}}\right) \\
& =\mathbb{P}\left(\inf \sigma\left(H_{L}\right) \leq \lambda+C L^{-1 / 2}\right),
\end{aligned}
$$

where $C \geq 1$ is some $L$-independent constant, and the equality in the last line relies on Lemma 4.3. The claim now follows from Lemma 5.6.

\section{Acknowledgment}

We thank Werner Kirsch for stimulating discussions and the German Research Council (Dfg) for partial financial support through Sfb/Tr 12 .

\section{References}

[AZ97] A. Altland and M. Zirnbauer, Nonstandard symmetry classes in mesoscopic normal-superconducting hybrid structures, Phys. Rev. B 55, 11421161 (1997).

[CL90] R. Carmona and J. Lacroix, Spectral theory of random Schrödinger operators, Birkhäuser, Boston, 1990.

[E12] A. Elgart, private communication, 2012.

[ESS12] A. Elgart, M. Shamis and S. Sodin, Localisation for non-monotone Schrödinger operators, preprint arXiv:1201.2211.

[G11] M. Gebert, Anderson localization for random block operators, Diploma thesis, LMU München, 2011.

[GK01] F. Germinet and A. Klein, Bootstrap multiscale analysis and localization in random media, Commun. Math. Phys. 222, 415-448 (2001).

[GK04] F. Germinet and A. Klein, A characterization of the Anderson metalinsulator transport transition, Duke Math. J. 124, 309-350 (2004).

[GK06] F. Germinet and A. Klein, New characterizations of the region of complete localization for random Schrödinger operators, J. Stat. Phys. 122, 73-94 (2006).

[K89] W. Kirsch, Random Schrödinger operators: a course. In Schrödinger Operators, H. Holden and A. Jensen (Eds.), Lecture Notes in Physics, vol. 345, Springer, Berlin, 1989, pp. 264-370.

[K08] W. Kirsch, An invitation to random Schrödinger operators, Panoramas et Synthèses 25, 1-119 (2008).

[KMM11] W. Kirsch, B. Metzger and P. Müller, Random block operators, J. Stat. Phys. 143, 1035-1054 (2011).

[KS80] H. Kunz and B. Souillard, Sur le spectre des opérateurs aux différences finies aléatoires, Commun. Math. Phys. 78, 201-246 (1980).

[PF92] L. A. Pastur and A. Figotin, Spectra of random and almost-periodic operators, Springer, Berlin, 1992. 
[T08] C. Tretter, Spectral theory of block operator matrices and applications, Imperial College Press, London, 2008.

\author{
Martin Gebert \\ Mathematisches Institut, Universität München, Theresienstraße 39, 80333 \\ München, Germany \\ e-mail: gebert@math.lmu.de \\ Peter Müller \\ Mathematisches Institut, Universität München, Theresienstraße 39, 80333 \\ München, Germany \\ e-mail: mueller@lmu.de
}

